\title{
(c) \\ PROLIFERATION OF MESENCHYMAL STEM CELLS IN CARBONATE APATITE-CHITOSAN SCAFFOLDS IN BONE TISSUE ENGINEERING TECHNIQUES
}

\begin{abstract}
Objectives: Chitosan is a popular bone graft material. However, chitosan also has a weakness for cell adhesion and the lack of sufficient bone formation capabilities. To improve it, we tried to develop a chitosan scaffolds that combine with carbonate apatite (CA), which has excellent biocompatibility properties of the tissues of the human body. We tried to find the most appropriate amount of Carbon Apatite (CA) to be combined with Chitosan scaffold $(\mathrm{ChSs})$ to produce a good scaffolds structure, as well as to evaluate CA-ChSs from the standpoint of cell proliferation using mesenchymal stem cells (MSCs).
\end{abstract}

Materials and Methods: Porous chitosan matrix was made by using the lyophilization technique. ChSs containing chitosan powder was made by the following procedure. 100, 150, 200, and $400 \mathrm{mg}$ of chitosan powder was dissolved in $5 \mathrm{ml}$ of $2 \%$ acetic acid at room temperature. ChSs containing 200 mg of chitosan powder was chosen to make CA-ChSs. Ultraviolet radiation was then performed for 2 hours. CA-ChSs structure was observed by a scanning electron microscope (SEM). Proliferation of MSCs in CA-ChSs evaluated at days $1,3,5$ and 7 .

Results: This study demonstrated that CA-ChSs containing $200 \mathrm{mg}$ of chitosan powder and $50 \mathrm{mg} \mathrm{CA}$ has a three-dimensional structure that is porous and attachment powder $\mathrm{CA}$ in the pores and absorbance values were increased from the examination day 1 until day 7. Cell proliferation using MSCs in CA-ChSs are better, as the absorbance value of CA-Chss with $50 \mathrm{mg} \mathrm{CA}$ content was significantly higher than ChSs. These findings also confirm that MSCs has good viability and biocompatibility in bone tissue engineering techniques.

Conclusions: Based on these results, it is expected CA-ChSs may be candidates for bone graft material in tissue engineering techniques.

Keywords: Chitosan, carbonic apetite, mesenchymal stem cell, scaffold.
Aqsa Sjuhada OKI ${ }^{1}$

Maretaningtias Dwi ARIANI ${ }^{2}$

ORCID IDs of the authors: A.S.O. 0000-0003-4427-2561 M.D.A. 0000-0002-7473-2015

\footnotetext{
${ }^{1}$ Department of Oral Biology, Faculty of Dental Medicine, Universitas Airlangga, Indonesia.

${ }^{2}$ Department of Prosthodontics, Faculty of Dental Medicine, Universitas Airlangga, Surabaya, Indonesia
}

How to Cite: Oki AS, Ariani MD. Proliferation of Mesenchymal Stem Cells in Carbonate Apatite-Chitosan Scaffolds in Bone Tissue Engineering Techniques. Cumhuriyet Dent J 2019;22:4

*Corresponding Author:

Department of Oral Biology, Faculty of Dental Medicine, Universitas Airlangga, Indonesia. 


\section{INTRODUCTION}

The need for bone repair has increased with increasing life expectancy. Bone defects caused by tooth extraction, trauma and other pathological conditions have limitations in spontaneous bone repair. In this regard, strategies for improving bone defects include autografts, allografts and xenografts. Autografts have become the ideal choice to fill the bone defects. ${ }^{1}$ However, this method has several significant disadvantages that may cause complications in the healing process, require additional surgical measures, cause pain to donors and limited supply of bone donors. On the other hand, allografts and xenografts have risks to immunogenic reactions and disease transmission from liquids and donor tissues. ${ }^{2}$ Given these weaknesses, it encourages increased use of alternative bone graft materials to develop the ideal bone graft material in tissue engineering techniques. $^{3}$

In tissue engineering techniques, there are three important pillars, namely cells, which play a role in the regeneration of new tissues; scaffolds, providing matrices for cells; and signaling molecules, which can enhance attachment, cell proliferation and differentiation. For the success of tissue engineering techniques, it is important to optimize the three pillars. ${ }^{4}$ Platelet-rich plasma have been developed as grafting material in bone regeneration, it resulted excellent primary stabilty and radiographic integration. ${ }^{5}$

Scaffolds play an important role in tissue engineering techniques to support bone regeneration. Currently, several natural and synthetic materials have been used to develop three-dimensional scaffolds that play an important role as artificial extracellular matrix, supporting cell proliferation and differentiation as well as maintaining its function. Particular attention has been given to scaffolds polymers for in vitro research on cell-scaffolds interactions and in vivo studies of bone graft materials. ${ }^{6}$

Chitosan is an interesting topic in the development of bone graft material because it has the desired characteristics with regard to its potential as a cell-scaffolds. Chitosan is an amino polysaccharide (poly-1,4-D-glucosamine), which is a deacetylation product of chitin. Chitosan is considered a good candidate for bone graft material because it has high biocompatibility and biodegradability, non-toxic and has good adsorption properties. Chitosan may increase proliferation and differentiation in osteoblast cell cultures. ${ }^{7}$ Chitosan has other useful characteristics, including bacteriostatic, hemostatic, anticholesterol and as enzymatic degradation. The properties of chitosan matrix, such as microstructure, crystallinity and mechanical strength, can be varied by changing the concentration, molecular weight and degrees of chitosan deacetylation. Furthermore, chitosan can be made into various forms and has been widely applied in the biomedical field. ${ }^{8}$

To make suitable scaffolds a bone graft material, current efforts are focused on improving mechanical strength and biological properties through the incorporation of bioceramic materials, such as carbonate apatite (CA). CA and other calcium phosphate $(\mathrm{CaP})$ are important for the repair of bone tissue because they have similar properties to minerals in bone. ${ }^{9}$ As already well known that the main content of bone inorganic material is CA which contains about $7 \%$ carbonate of total weight. CA is easily soluble in acidic conditions and has thermodynamic properties in neutral conditions. Therefore, $\mathrm{CA}$ is expected to be a substitute for ideal bone material, which has both osseoconductive and bioresorbable properties. ${ }^{10}$

The specific properties of chitosan and CA can be combined to create a carbonate apatitechitosan scaffolds (CA-ChSs) as a new bone graft material with unique structural and mechanical properties. CA-Chss can act as bioactive materials that can affect cell proliferation and differentiation. In addition to scaffolds that play an important role in bone tissue engineering techniques, the selection and use of cells also affects the success of tissue engineering techniques. A previous study has found that CA-ChSs is biocompatible and supports mouse osteoblast-like cell proliferation (MC3T3E1). ${ }^{11}$ However, there has never been a mesenchymal stem cell culture (MSCs) in CAChSs. 
Mesenchymal stem cells have been proven to be the most promising craniomaxillofacial application for a therapeutic support in bone engineering, as compared to the use of conventional antologous and allogenic bone grafts. $^{12}$

Stem cells are recommended as a treatment option to repair tissue damage, including bone defects. In this case, the MSCs present in the bone marrow receive special attention because of their differentiated ability to be bone, nerve, carotid and muscle tissue. ${ }^{13}$

Therefore, it is necessary to conduct a study to determine the interaction of MSCs in CA-ChSs and to evaluate the proliferation and differentiation of MSCs in scaffolds.

\section{MATERIALS AND METHODS}

This study was cleared by the faculty's Ethical Clearance Commission. The porous chitosan matrix was prepared using lyophilization technique. ChSs containing 100, 150, 200, and 400 $\mathrm{mg}$ of chitosan powder were prepared by the following procedure. One hundred, 150, 200, and $400 \mathrm{mg}$ of chitosan powder $(98.7 \%$ deacetylation grade, YSK, Japan) were dissolved in $5 \mathrm{ml}$ of $2 \%$ acetic acid at room temperature, shaken for $15 \mathrm{~min}$, then neutralized with $15 \mathrm{ml} 0.1 \mathrm{M} \mathrm{NaOH}$ solution After centrifugation At $1500 \mathrm{rpm}$ for 10 minutes, the excess water is removed, then the chitosan gel is packed into the mold (diameter: $5 \mathrm{~mm}$, height: 2 $\mathrm{mm}$ ). Molds were frozen at $-80^{\circ} \mathrm{C}$ for 2 hours and dried in a dry-freeze machine at $-54^{\circ} \mathrm{C}$ for 24 hours. To make CA-ChSs, ChSs were selected containing $200 \mathrm{mg}$ of chitosan powder. After neutralization, $50,100,200$ and $300 \mathrm{mg}$ of $0.06 \mathrm{M} \mathrm{CA}$ were added to the chitosan gel containing $200 \mathrm{mg}$ of chitosan powder.

When the process of making CA-ChSs, acetic acid solution was used to dissolve chitosan powder and then neutralized with $\mathrm{NaOH}$ solution. To remove alkaline salts, ions or some toxic substances in CA-ChSs, desalinations were carried on. Desalination was performed by the following procedure, $25 \mathrm{~cm}$ plastic cellulose was prepared and $100 \mathrm{ml}$ of distilled water was added. ChSs and CA-ChSs are inserted into cellulose plastic, then placed under tap water for 24 hours and frozen at - $80^{\circ} \mathrm{C}$ for 2 hours. The scaffolding was moved to a freeze dried machine at $-54^{\circ} \mathrm{C}$ for 24 hours. Furthermore, ultraviolet radiation in $\mathrm{ChSs}$ and CAChSs for 2 hours. The sample is ready to be tested in cell culture.

Scaffolds are randomly selected and macroscopic photos of scaffolds are taken using a digital camera. Microscopic structures and porosity scaffolds were observed using SEM (3D Microscope VE-8800, Keyence, Japan).

Prior to the testing, chitosan and powdered CA samples were mixed with potassium bromide $(\mathrm{KBr})$ then were grinded using mortal to remove the scattering effect of large crystals. The delicate and uniform samples are mechanically compressed to form a translucent pellets that can be passed through the infrared spectrum. The FTIR test produces a transmission graph (\%) of the wave number $\left(\mathrm{cm}^{-1}\right)$.

The compressive strength test is performed by using a sloped CA-ChSs sample which is topped and the bottom is smoothed with sandpaper, the sample side is measured using a sliding range. The sample is placed on the press machine compression unit, then the engine is turned on and set the speed and style to be measured. Load cell slowly lowered then stopped and recorded the amount of force obtained.

Culture MSCs (JCRB Cell Bank, Japan) in the form of cell-line grown in dish. After confluent, the culture was harvested using trypsine versene solution. The yields were replanted in a DMEM medium containing $10 \%$ bovine serum albumin, $2 \%$ penicillin / streptomycin, incubated in $5 \% \mathrm{CO}_{2}$ at $37^{\circ} \mathrm{C}$. Media is replaced every $3-4$ days and done sub culture every 7 days. Then the cell is moved in a small bottle and made with a density of 2 x 104 cells $/ \mathrm{ml}$, the cell is ready to be used for sample testing.

This test is carried out according to the recommended protocol standard for the MTT essay. Cell proliferation in scaffolds is examined using an MTT essay. $20 \mu \mathrm{l}$ cell suspension with a density of 2 x 104 cells was added to ChSs and CAChSs placed in a 24-well tissue culture plate. After 2 hours, $980 \mu 1$ DMEM was added to each well. Then the cells were incubated in the incubator $\left(\mathrm{CO}_{2}\right.$ 
$5 \%$ at $37^{\circ} \mathrm{C}$ ). Media culture is replaced every 3 days.

On observations of days 1, 3, 5 and 7, ChSs and CA-ChSs were washed using PBS, incubated for $2 \mathrm{~h}$ in $500 \mu \mathrm{l}$ of medium culture containing 50 $\mu$ of MTT reagent, the well was introduced into the incubator $\left(\mathrm{CO}_{2} \quad 5 \%\right.$ at $\left.37^{\circ} \mathrm{C}\right)$. The media supernatant $(110 \mu \mathrm{l})$ was transferred to a 96-well culture plate and the absorbance was measured using a microplate reader with a wavelength of 450 $\mathrm{nm}$. The results obtained are expressed in optical density (absorbent). Large absorbents of each well indicate the amount of cell proliferation in a media culture.

Statistical analysis was performed using SPSS. The result of the measurement is tabulated according to each group, then tested one way Anova statistic with 5\% significance level. If there is a meaningful difference then proceed with LSD test. This study was conducted in accordance to ethical clearance, which was declared by the faculty ethical committee.

\section{RESULTS}

The macroscopic photos of ChSs are shown in Figure1. ChSs containing 100 and $150 \mathrm{mg}$ of chitosan powder have fragile and fragile properties (Figs 1A and 1B). In contrast, ChSs containing 200 and $400 \mathrm{mg}$ of chitosan powder have good form and handling properties (Figs 1C and 1D).
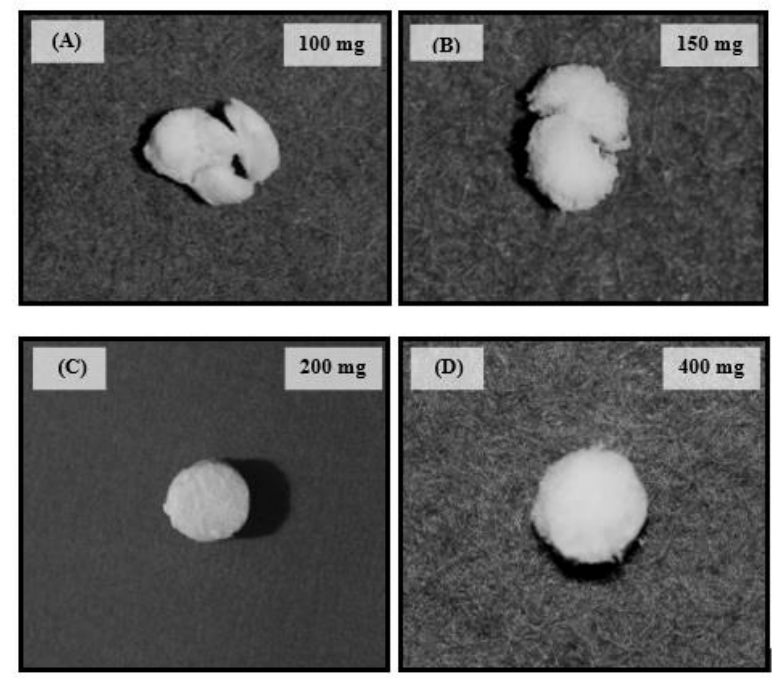

Figure 1. Macroscopic photos of $\mathrm{ChSs}$ with different amounts of chitosan powder (A) $100 \mathrm{mg}$; (B) $150 \mathrm{mg}$; (C) $200 \mathrm{mg}$ and (D) 400 mg.
The results of the test using SEM (Fig. 2A), showed that ChSs with $200 \mathrm{mg}$ of chitosan powder had a three-dimensional structure with many pores. On the other hand, ChSs with $400 \mathrm{mg}$ of chitosan powder have a three-dimensional structure with little pores (Fig. 2B). These results indicate that ChSs with $200 \mathrm{mg}$ chitosan powder is a scaffold that has good handling.
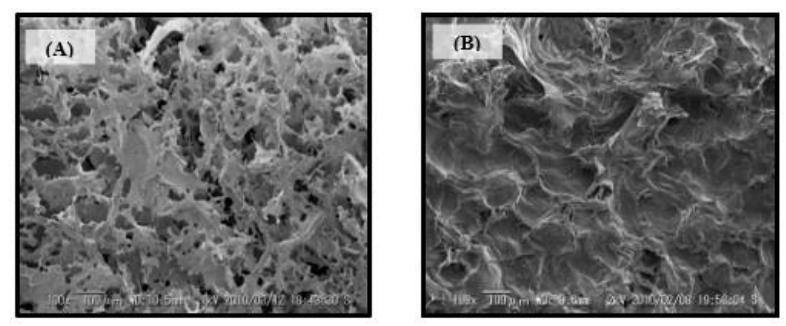

Figure 2. Examination of SEM ChSs with content (A) $200 \mathrm{mg}$ and (B) $400 \mathrm{mg}$ chitosan powder.

Figure 3 shows that $\mathrm{CA}-\mathrm{ChSs}$ made with different amounts of CA powder have a threedimensional structure with many pores. The pore size ranges from 50-200 mm. In general, CA-ChSs with $50 \mathrm{mg}$ of $\mathrm{CA}$ has a three-dimensional structure with the most CA bonds in its pores (Fig $3 \mathrm{~A})$.
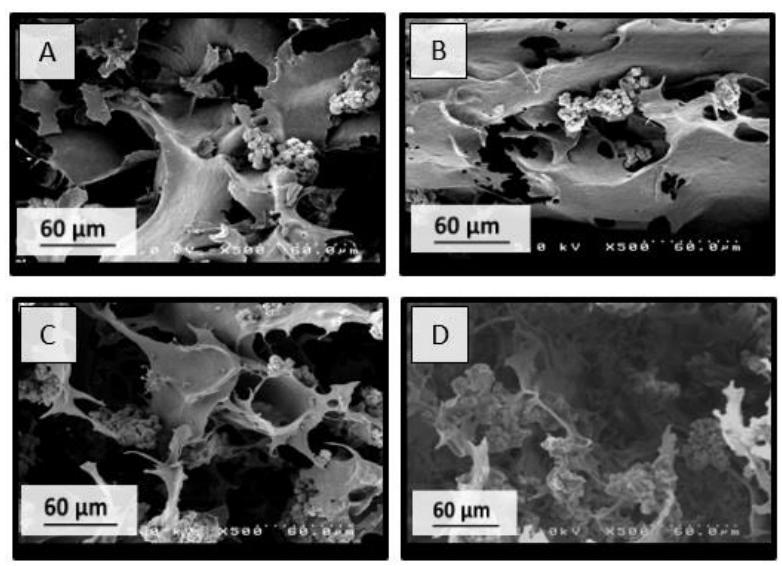

Figure 3. SEM CA-ChSs examinations with different amount of CA powder (A) $50 \mathrm{mg}$, (B) $100 \mathrm{mg}$, (C) $200 \mathrm{mg}$ and (D) $300 \mathrm{mg}$.

The FT-IR CA spectrum shows the peak of transmittance for phosphate groups at 1000-1100 $\mathrm{cm}-1$ and 500-600 cm-1, carbonate groups at 1400 $1500 \mathrm{~cm}-1$, and hydroxyl groups at $3500-3600 \mathrm{~cm}-$ 1. On the other hand, the HA spectrum does not have a peak on the carbonate group, while the peak of the phosphate and hydroxyl groups can be observed (Fig 4). 


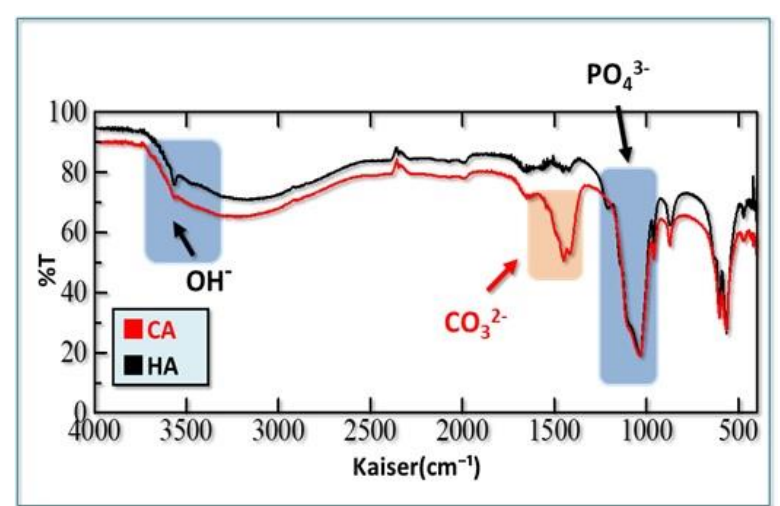

Figure 4. FT-IR spectrum. CA (red) and HA (black) synthesized in this study.

The absorbance value of CA-Chss with $50 \mathrm{mg}$ CA content was significantly higher than ChSs (Fig $5)$.

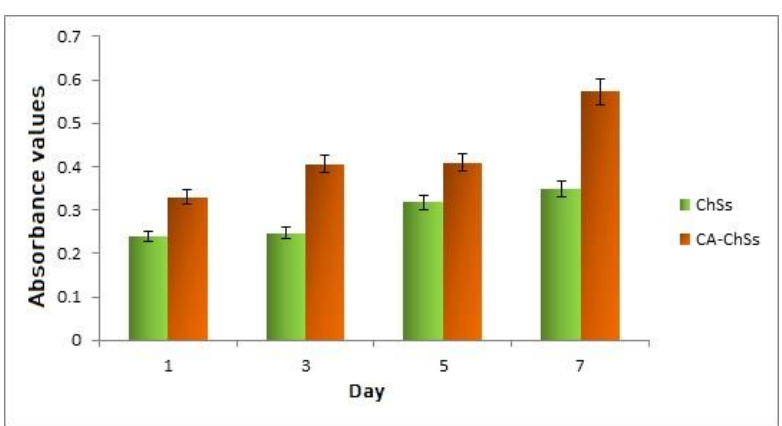

Figure 5. Proliferation test results of ChSs dan CA-ChSs.

Cell proliferation test results in $\mathrm{ChSs}$ and CA-ChSs are shown in Table 1.

Table 1. The absorbance value of ChSs and CA-ChSs $(\mathrm{p}<0.05)$.

\begin{tabular}{lcccc}
\hline Sampel & Day $\mathbf{1}$ & Day $\mathbf{3}$ & Day 5 & Day 7 \\
\hline ChSs & $0.239 \pm 0.02$ & $0.247 \pm 0.06$ & $0.318 \pm 0.05$ & $0.348 \pm 0.09$ \\
CA-ChSs & $0.330 \pm 0.04$ & $0.406 \pm 0.07$ & $0.409 \pm 0.08$ & $0.572 \pm 1.01$ \\
\hline
\end{tabular}

\section{DISCUSSION}

In bone tissue engineering techniques, scaffolds have an important role in supporting bone regeneration. To date, much effort has been made to develop a scaffold with a three-dimensional structure that provides the necessary support as an extracellular matrix, allowing cells to proliferate and differentiate. A good scaffold should be nontoxic, biodegradable, biocompatible and has high porosity and mechanical properties. ${ }^{14}$

In the present study, we developed a scaffold that combines chitosan with CA. First, an evaluation of the amount of chitosan powder used in the preparation of ChSs to determine the optimal concentration of chitosan powder using SEM examination. Based on SEM, ChSs with $200 \mathrm{mg}$ of chitosan powder have good handling properties and three-dimensional structure with pore size about 50-200 $\mu \mathrm{m}$. From the results of this examination, it can be concluded that the optimal concentration of chitosan powder that can be used in the manufacture of ChSs is $200 \mathrm{mg} .{ }^{15}$

Current efforts are focused on improving the ability of ChSs in bone formation by combining ChSs with a calcium phosphate substance, such as hydroxyapatite (HA), $\beta$-tricalcium phosphate $(\beta$ TCP) and carbonate apatite (CA). ${ }^{7} \mathrm{HA}$ and $\beta$-TCP have some disadvantages such as degradation or dissolution rate that is difficult to predict. HA made by sintering process with high temperature has non-degradable properties. However, it has been reported that the combination of calcium hydroxyapatite (IP / CHA) has systemic pores and almost all pores are interconnected and have good bone-building abilities in in vivo studies. On the other hand, the crystallinity of $\beta$-TCP is too low, so in some cases it will be absorbed before it gets enough new bone formation. Among calcium phosphate, it has been reported that CA has an appropriate absorption time and excellent bonebuilding ability. In this study, selected and combined chitosan which is a biodegradable material and CA to obtain an appropriate period of absorbance and improve bone-building ability. ${ }^{16}$

The properties of a scaffold are also influenced by fabrication techniques. Several techniques have been developed to create scaffolds, including solvent-casting techniques, sol-gel techniques and lyophilization techniques. However, it has been reported that in solventcasting techniques, the presence of pyrogen salts may affect the cell due to loss of water-soluble biomolecules and non-uniform deformation induction. In sol-gel technique, there are some disadvantages such as difficulty in proper control 
of pore structure and hydrolysis and condensation process. In this study, lyophilization techniques were adopted to make scaffolds, since the technique is simpler and easier than any other method described previously and by using lyophilization techniques, the porous nature of CAChSs can be obtained from the freeze dried process. ${ }^{17}$

Today, one promising network engineering strategy is to use a scaffold that has a threedimensional porous structure to facilitate regeneration of bone tissue. As a scaffold for tissue engineering techniques should have a porous structure with pore size ranging from $40-300 \mu \mathrm{m}$ to allow for migration and cell vascularization and growth factor. In this study, CA-ChSs produced good handling properties and had a threedimensional porous structure with a pore size of about $50-200 \mu \mathrm{m} .{ }^{18}$

When deciding to use CA-ChSs in clinical applications, for example in cases of bone augmentation of buccal defects from implant treatment or to fill the bone socket after tooth extraction, the CA-ChSs should be sterilized before use. Since some interventions on oral tissue may lead to bleeding, perilesional edema, and mucosal atrophy, the sterilization process was extremely required to avoid any possible complications during the procedures. It is also expected to improve chronic traumatisms in periodontal diseases. ${ }^{19,20}$ There are several methods of sterilization that have been in use, including gamma irradiation, steam autoclaving, ethylene oxide and radio frequency plasma glow discharge. Since CA-ChSs is an organic polymer composite, gamma irradiation is a suitable sterilization method for CA-ChSs to maintain its chemical structure. ${ }^{15}$

After the development of a scaffold that has a porous structure, then the selection of the right cells can also affect the success of network engineering techniques. In this study, MSCs cells were cultured on CA-ChSs for cell proliferation measurements, because in the application of bone tissue engineering techniques, stem cells were usually used to confirm their responsibility for bone formation. ${ }^{13,21}$

From cell proliferation test results, indicating that by combining $\mathrm{ChSs}$ and $\mathrm{CA}$ powders, cell proliferation capability was higher than in $\mathrm{ChSs}$. It can be said that with the addition of CA powder into $\mathrm{ChSs}$ it can increase the surface area of the scaffold so that the cell can attach more. Furthermore, CA-ChSs made from $200 \mathrm{mg}$ of chitosan powder plus $50 \mathrm{mg}$ of CA powder showed cell proliferation ability significantly higher than other groups. ${ }^{14,22}$

Furthermore, a histologic study of CA-ChSs is required to ensure its property in bone regeneration. Based on the results of this study, it can be concluded that CA-ChSs has a porous threedimensional structure that is interconnected with each other, has good handling properties as well as the ability to support MSCs proliferation so that CA-ChSs is very likely to be used as material for bone tissue engineering. ${ }^{15}$

\section{CONCLUSSIONS}

From this study it can be concluded that $200 \mathrm{mg}$ of $\mathrm{CA}$ is the most effective amount to be combined with ChSS to produce an excellent scaffold on the standpoint of three dimensional structure with little pores. CA-ChSs developed from $200 \mathrm{mg}$ of chitosan plus $50 \mathrm{mg}$ of CA powder demonstrated the highest ability of cell proliferation among the groups.

\section{ACKNOWLEDGEMENTS}

None

\section{CONFLICT OF INTEREST STATEMENT}

None

\section{REFERENCES}

1. Riezzo I, Pascalo N, La Russo R, Liso A, Salerno M, Turillazzi E. Donor selection for allogenic hemopoietic stem cell transplantation: clinical and ethical consideration. Stem Cell Int 2017; 2017:1-11.

2. Topkaya T, Somaz MY, Dundar S, Eltas A. Numerical analysis of the effect of implant geometry to stress distributions of the three different commercial dental implant system. Cumhuriyet Dent J 2014; 18:1724.

3. Altin KT, Ekci ES, Gurdogan EB, Senoz D. A general outlook to regenerate pulp therapy. Cumhuriyet Dent J 2016; 19:238-246.

4. Hellen E, Fuchs E. Tissue patterning and cellular mechanics. J Cell Biol 2015; 211:219-231.

5. Inchingolo $\mathrm{F}$, Tatullo $\mathrm{M}$, Marrelli $\mathrm{M}$, Inchingolo AD, Dipalma G, Flace P, Girolamo F, Tarullo A, Laino 
L, Sabatini R, Abbinate A, Cagiano R. Regenerative surgery performed with platelet-rich plasma used in sinus lift elevation before dental implat surgery: an useful aid in healing and regeneration of bone tissue. Eur Rev Med Pharmacol Sci 2012; 16:1222-1236.

6. Ghassemi T, Shahroodi A, Ebrahimzadeh MH, Mousavian A, Movaffagh J, Moradi A. Current concepts in scaffolding for bone tissue engineering. Arch Bone Jt Surg 2018; 6:90-99.

7. Kazusko SD, Riccio C, Goulart M, Bumgardner J, Jing XL, Konofaos P. Chitosan as a bone scaffold biomaterial. J Craniofac Surg 2018; 29:1788-1793.

8. Srividya S, Sastry TP, Jeevith D, Samiksha N. Synthesis and characterization of a novel bone graft material containing biphasic calcium phosphate and chitosan fortified with aloe vera. Int J Drug Regul Aff 2014; 2: 85-90.

9. Mishra CB, Kumari S, Angeli A, Bua S, Tiwari M, Claudiu T. Discovery of benzenesulfonamide derivatives as carbonic anhidrase inhibitors with effective anticonvulsant action: design, synthesis, pharmacological evaluation. J Med Chem 2018; 61: 3151-3165.

10.Zang S, Zhu L, Luo K, Mu R, Chen F, Wei X, Yan X, Han B, Shi X, Wang Q, Jin L. Chitosan composite scaffold combined with bone marrow-derived mesenchymal stem cells for bone regeneration: in vitro and in vivo evaluation. Oncotarget 2017; 8: 110890110-903.

11. Debnath, T. Ghosh, S. Potlapuvu, US. Kona, L, Kamaraju, SR. Sarkar, S. Gaddam, S. Chelluri, LK. Proliferation and Differentiation Potential of Human Adipose-Derived Stem Cells Grown on Cytosan Hydrogel. Plos One. 2015; 10(3): e0120803.

12. Paduano F, Marrelli M, Amantea M, Rengo C, Rengo S, Goldgerg M, Spagnuolo G, Tatullo M. Adipose tissue as a strategic source of mesenchymal stem cells in bone regeneration: A topical review on the most promising craniomaxillofacial applications. Int $\mathbf{J}$ Mol Sci 2017; 18: 1-12.

13.Zang S, Jin L, Kang S, Hu X, Wang M, Wang J, Chen B, Peng B, Wang Q. Periodontal wound healing by transplantation of jaw bone marrow-derived mesenchimal stem cells in chitosan/anorganic bovine bone carrier into one-wall infrabony defects in beagles. J Periodontol 2016; 87: 971-981.

14. Soeroso Y, Bachtiar E, Bachtiar BM, Sulijaya B, Prayitno SW. The prospect of chitosan on the osteogenesis of periodontal ligament stem cells. J Int Dent Med Res 2016; 5: 93-97.

15. Seshandri S, Thotapalli S, Kumar BS. Synthesis and characterization of a novel bone graft material using biphasic calcium phosphate casein chitosan with the extracts of coriandrum sativum. Int J Pharm Pharm Sci 2014; 6: 358-361.

16. Van De Graaf GMM, De Zoppa ALV, Moreira RC, Maestrelli SC, Marques RFC, Campos MGN. Morphological and mechanical characterization of chitosan-calcium phosphate composite for potential application as bone-graft substitutes. Res Biomed Eng 2015; 31: 334-342.

17. Oki AS, Bimarahmanda ME, Rahardjo MB. Increased number of fibroblasts and neovascularization after tooth extraction in wistar rats with moderateintensity continuous exercise. J Int Dent Med Res 2018; 11: 840-845.

18. Chatzipetros E, Christapoulos P, Donta C, Tosius KI, Tsiambas E, Tsiourvas D, Kalogirou E-M, Tsiklakis K. Application of nano-hydroxyapatite/chitosan scaffolds on rat calvarial critical-sized defects: a pilot study. Med Patol Oral Cir Bucal 2018; 23: e625-e632. 19. Inchingolo F, Tatullo M, Abenavoli FM, Marrelli M, Inchingolo AD, Palladino A, Inchingolo AM, Dipalma G. Oral piercing and oral disease: $A$ short time retrospective study. Int J Med Sci 2011; 8: 649-652.

20. Inchingolo F, Tatullo M, Abenavoli FM, Marrelli M, Inchingolo $\mathrm{AD}$, Inchingolo AM, Dipalma G. NonHodgkin lymphoma affecting the tongue: unusual intraoral location. Head Neck Oncol 2011; 3:1-5.

21. Oki AS, Farhana N, Yuliati. The effect of aerobic and anaerobic interval exercise on the proliferation phase of wound healing in tooth extraction of Rattus novergicus. Acta Med Philipp 2019; 53: 417-422.

22. Irmawati A, Giffari FZ, Oki AS. The effect of moderate exercise on vascular endothelial growth factor expression during tooth socket wound healing after tooth extraction. J Postgrad Med Inst 2018; 32: 19-23. 